\title{
Docetaxel Nanoparticle CPC634
}

National Cancer Institute

\section{Source}

National Cancer Institute. Docetaxel Nanoparticle CPC634. NCI Thesaurus. Code C121961.

A polymeric nanoparticle (PNP) formulation containing the poorly water-soluble taxane docetaxel, a semi-synthetic analogue of paclitaxel, with antineoplastic activity. Upon intravenous administration of the docetaxel nanoparticle CPC634, the nanoparticles are able to accumulate at the tumor site due to the unique characteristics of the tumor's vasculature, while avoiding normal, healthy tissue. In turn, docetaxel is released locally at the target tumor site, binds specifically to the beta-tubulin subunit of the microtubule, stabilizing tubulin and inhibiting microtubule disassembly, which results in cell-cycle arrest at the G2/M phase, thereby preventing cell proliferation. This agent also inhibits proang iogenic factors such as vascular endothelial growth factor (VEGF) and induces various mediators of the inflammatory response. Compared to docetaxel alone, this formulation may enhance stability and improve delivery, thereby increasing docetaxel's efficacy while avoiding systemic exposure, which minimizes its toxicity. 